\title{
Effect of Cooperative and Selection Relaying Schemes on Multiuser Diversity in Downlink Cellular Systems with Relays
}

\author{
Min Suk Kang, Bang Chul Jung, and Dan Keun Sung
}

\begin{abstract}
In this paper, we investigate the effect of cooperative and selection relaying schemes on multiuser diversity in downlink cellular systems with fixed relay stations (RSs). Each mobile station (MS) is either directly connected to a base station (BS) and/or connected to a relay station. We first derive closed-form solutions or upper-bound of the ergodic and outage capacities of four different downlink data relaying schemes: A direct scheme, a relay scheme, a selection scheme, and a cooperative scheme. The selection scheme selects the best access link between the BS and an MS. For all schemes, the capacity of the BS-RS link is assumed to be always larger than that of RS-MS link. Half-duplex channel use and repetition based relaying schemes are assumed for relaying operations. We also analyze the system capacity in a multiuser diversity environment in which a maximum signal-to-noise ratio (SNR) scheduler is used at a base station. The result shows that the selection scheme outperforms the other three schemes in terms of link ergodic capacity, link outage capacity, and system ergodic capacity. Furthermore, our results show that cooperative and selection diversity techniques limit the performance gain that could have been achieved by the multiuser diversity technique.
\end{abstract}

Index Terms: Cellular systems, cooperative diversity, multiuser diversity, opportunistic scheduling, relays, selection diversity.

\section{INTRODUCTION}

In wireless communications, wireless channels are usually varying in time and frequency. Since there is a rather high probability that the channel is in a deep fade for mobile users, we need some diversity techniques in order to compensate for this channel impairment. This motivates us to investigate various $d i$ versity techniques which can improve the wireless link capacity of mobile communication systems. Before the concept of diversity, channel fading always degrades channel capacity; however, utilizing the concept of diversity, we can counteract the adverse effect of the channel fading and improve the reliability of communication in fading channels. In conventional wireless communication systems, diversity techniques using antenna arrays or RAKE receivers can effectively combat against fading [1], [2]. These techniques achieve diversity gain from the channel fading over time, frequency, and space. In addition to these

Manuscript received December 16, 2007.

M. S. Kang and B. C. Jung are with KAIST Institute for Information Technology Convergence, Daejeon, 305-701, Korea, email: \{minsuk.kang, dksung @ kaist.ac.kr.

D. K. Sung is with the School of Electrical Engineering and Computer Science, KAIST, Daejeon, 305-701, Korea. email: dksung@ee.kaist.ac.kr.

This paper was presented in part at IEEE International Symposium on Communications and Information Technologies (ISCIT), Sydney, Australia, October 2007.

This work was supported by the BrOMA IT Research Center. conventional diversity techniques, diversity gain can be also achieved through cooperation among geographically distributed nodes or terminals. This cooperative diversity was first studied in [3], [4] and low-complexity cooperative diversity protocols were proposed and analyzed in [5], where two user cooperation strategies were studied in a single relay node environment. Diversity gains can be achieved through relays. Studies on fixed or nomadic relays have been done [6]-[8] and they are mainly aimed to the users near cell-boundary or in the dead spots. If multiple relays are available in transmission, we can achieve selection diversity by selecting one or multiple relays based on channel state information (CSI) [9].

If we utilize multiuser fading channels, we can improve system performance. Knopp and Humblet [10] first introduced multiuser diversity as a means to provide diversity against channel fading in multiuser communication systems. The performance gain of multiuser diversity increases as the number of active users in the system becomes large [11]. When a base station (BS) selects one user with the maximum signal-to-noise ratio (SNR) value among multiple users, it has a higher probability to capture users with higher SNR values as the number of users increases. As high data-rate demands for internet users increase rapidly, this multiuser diversity concept has been widely accepted and deployed in many next generation mobile communication systems, such as 3rd generation partnership project (3GPP) long term evolution (LTE), IEEE 802.16 mobile WiMax, or 3rd generation partnership project 2 (3GPP2) ultra mobile broadband (UMB) to maximize the system capacity. Typical scheduling schemes for achieving multiuser diversity include maximum SNR and proportional fairness (PF) schemes.

While the conventional diversity techniques have been used to average out the effect of channel fading, multiuser diversity exploits the effect of multiuser channel fading. We can think of the conventional diversities as link diversity techniques in a sense that they only consider the diversity within the channel fading of one link communication; however, multiuser diversity can be considered as a system diversity technique because it considers multiple channel links and exploits the diversity among them. The link diversity techniques are known to be very effective in one linke environment. For example, space-time block code (STBC), known as the most effective spatial diversity technique, greatly increases the channel capacity and outage capacity in multiple antenna systems [12]. However, the effect of multiuser diversity on STBC was investigated and various aspects related to the combined use of spatial diversity and multiuser diversity in a mobile network was studied [13]. In [13], spatial diversity is shown to eliminate the peaks of the Rayleigh 
fading channel, thus limiting the performance gain that could have been achieved by the multiuser diversity mechanism. It is due to the fact that spatial diversity lessens the variation of the channel and the reduced variation makes the channel less fluctuated and it results in reducing the multiuser diversity gain.

Cooperative and selection diversity techniques in cellular systems with relays have been studied in several papers. A dual-hop transmission with fixed gain relays was investigated in terms of outage probability and average error probability [14]. More recently, a new relaying scheme that exploits multiuser diversity in multihop networks was proposed in [15], [16], in which multiuser diversity is exploited in each hop by selecting the next hop relay based on the instantaneous channel quality. However, most part of their studies relied on simulation results and mathematical analysis on multiuser diversity and relay schemes was very limited. In [17], cooperative diversity and multiuser diversity in cooperative relay networks were mathematically analyzed. The result shows that both cooperative diversity and multiuser diversity are exploited in a system environment and the diversity increases as the number of source/destination pairs increases. However, the analysis is based on ad-hoc networks which have many source/destination pairs, and, thus, it is not suitable for cellular systems.

There have been no rigorous mathematical analysis on the effect of multiuser diversity in cellular system with relays. Moreover, the effect of cooperative and selection diversity techniques on the multiuser diversity gain has not been fully understood yet. In this paper, we mathematically analyze the ergodic capacity and the outage capacity in a single-link, multiuser environment. The analysis highlights the effect of multiuser diversity in various relay schemes. We investigate the effect of selection diversity and cooperative diversity on multiuser diversity in a downlink cellular system with multiple fixed relays.

The rest of this paper is organized as follows. In Section II, we introduce a system and channel model under consideration. In Section III, we analyze the performance of downlink capacity in terms of ergodic capacity and outage capacity. In Section IV, the performance of a multiuser system with relays is analyzed. Moreover, the effect of cooperative and selection diversities on multiuser diversity is also characterized by investigating the effect of variance of each transmit scheme. Finally, conclusions are presented in Section V.

\section{SYSTEM AND CHANNEL MODEL}

Fig. 1 shows a cellular downlink system with one BS located at the center of a cell, $R$ relay stations (RSs), and $N$ mobile stations (MSs). RSs do not have their own messages and are soley intended to assist the MSs and BS to communicate with one another. We assume that the RSs are nomadic or fixed and are operated in half-duplex mode, i.e., the RSs cannot transmit and receive messages simultaneously. We also assume that at a given time each MS communicates with the BS through one RS. That is, choosing one of the multiple RSs is not allowed to all MSs and for a specific time only one RS can be assigned to a MS. This assumption is made to simplify the mathematical model and show the result. Extension to multiple RSs for an MS can be possible by assigning more than one independent relay path to

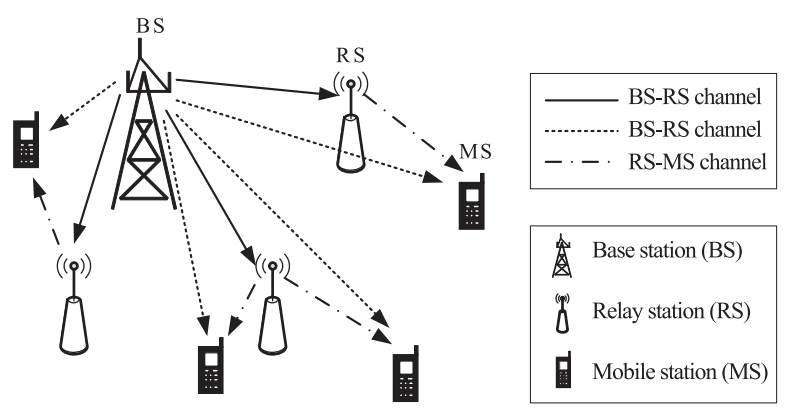

Fig. 1. System model.

the MS; however, the result would follow the similar tendency.

There are three different types of wireless channels in the model: A channel between a BS and an RS (BS-RS channel), a channel between a BS and an MS (BS-MS channel), and a channel between an RS and an MS (RS-MS channel). The capacity of BS-RS channels is assumed to be always better than that of RS-MS channels so that the capacity of a channel between the BS and an MS via an RS is always limited by the capacity of the channel between the RS and the MS. This assumption is reasonable for typical cellular systems with relays since relays are generally set up using reliable wired connection or at the place to support line-of-sight (LOS) to the BS. We assume that both the BS-MS and RS-MS channels follow Rayleigh distributions, and they are independent of each other. We also assume that all users have an identical channel environment for mathematical simplicity. Other-cell interference is also neglected in this single-cell mathematical analysis model.

We consider the following four downlink data relaying schemes: A direct scheme, a relay scheme, a selection scheme, and a cooperative scheme. The schemes are simple and practical relaying schemes that can be utilized in the system of Fig. 1. The four schemes have low system complexity; however, the amount of required feedback for each scheme is different. In a single user case, only the selection scheme requires the feedback of BS-MS and RS-MS channels to select the better path while the other three schemes do not require any feedback information. On the other hand, in a multiuser case, the selection scheme and the cooperative scheme require the feedback of BS-MS and RSMS channels for every MS in the cell while the direct scheme and the relay scheme only require the feedback of BS-MS channel and RS-MS channel, respectively, of every MS in the cell. In practice, since the feedback information is sent through uplink resources, it is hard to quantitatively measure the effect of the amount of the feedback information on downlink capacity. Thus, in this paper, we neglect this feedback overhead in the downlink system capacity.

\section{A. Direct Scheme}

In the direct scheme, an MS communicates only with a serving BS directly without a relay system, as shown in Fig. 2(a).

The system is considered as a conventional cellular system without relays. The signal received at the $i$ th user is given by

$$
y_{i}=h_{d, i} s_{i}+n_{i}, \quad(1 \leq i \leq N)
$$




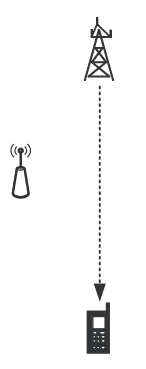

(a) Direct scheme

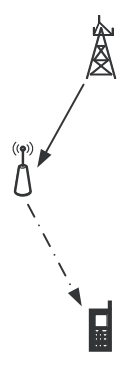

(b) Relay scheme

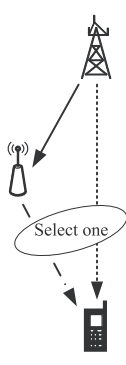

(c) Selection scheme

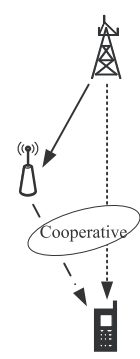

(d) Cooperative scheme
Fig. 2. Transmission for four different schemes.

where $h_{d, i}$ and $n_{i}$ represent the BS-MS channel coefficient and the thermal noise at the $i$ th user, respectively. Since the BSMS channel link is assumed to be Rayleigh distributed, $h_{d, i} \sim$ $C N\left(0, \mu_{d, i}^{2}\right)$, and $n_{i} \sim C N\left(0, N_{0}\right)$. The signal transmitted from the BS, $s_{i}$, has its transmit power constraint, $\mathbf{E}\left[\left|s_{i}\right|^{2}\right]=$ $P$. The term $\mu_{d, i}^{2}$ indicates the channel gain between the $\mathrm{BS}$ and the $i$ th MS, and it is assumed to be identical regardless of MSs as noted before. Thus, we remove the user index $i$ from now on. Since we assume the MS has the information of the channel $h_{d}$, the received signal-to-noise ratio (SNR) of a user is given by $\gamma \triangleq\left|h_{d}\right|^{2} P / N_{0}$. Thus, the probability density function (PDF) of the received SNR of the $i$ th user is given by

$$
f(\gamma)=\frac{1}{\rho \mu_{d}} e^{-\frac{\gamma}{\rho \mu_{d}}}
$$

where $\rho=P / N_{0}$ denotes the SNR at the transmitter (BS), i.e., the transmitted signal-to-noise ratio.

\section{B. Relay Scheme}

In the relay scheme, an MS communicates only with its only assigned RS, and the communication link between the BS and MS is assumed to be blocked, as shown in Fig. 2(b). In this system, each MS communicates with the BS via one RS. In this scheme, due to the half-duplex nature of a relay transceiver, the transmission time should be divided into two phases: Phase-I for receiving data from the BS at the RS, and Phase-II for sending the data from the RS to the MS. The ratio of time durations for Phase-I and Phase-II can be generally different for different relaying technologies and the capacity of the relaying system can be optimized by adaptively adjusting the ratio of the two phases [18]. However, in many promising mobile systems which adopt relay stations such as IEEE $802.16 \mathrm{j}$, the time slots for relay transmission and reception are assumed to be fixed since its ease of implementation and low complexity [19]. Thus, the time durations for two phases are assumed to be fixed and be the same in this paper. The signal received at user $i$ is given by

$$
\begin{aligned}
& y_{i, 1}=n_{i, 1}, \quad \text { in Phase I, } \\
& y_{i, 2}=h_{r, i} s_{i}+n_{i, 2} \text {, in Phase II }
\end{aligned}
$$

where $h_{r}$ and $n_{i}$ represent the RS-MS channel coefficient and the thermal noise at the $i$ th user, respectively. The transmit power of an RS is assumed to be the same as that of a BS. If we want to assign different transmit power to the RS, it can be done by adjusting the average channel gain between the RS and the MS, $\mu_{r} . y_{i, j}(i \in N, j \in\{1,2\})$ represents the received signal of the $i$ th user at Phase- $j$. Since the RS-MS channel link is also assumed to be Rayleigh distributed, $h_{r, i} \sim C N\left(0, \mu_{r, i}^{2}\right)$, $n_{i} \sim C N\left(0, N_{0}\right)$. The received SNR of the $i$ th user at Phase$I I$ is given by $\gamma_{r} \triangleq\left|h_{r}\right|^{2} P / N_{0}$. Thus, the PDF $f_{r}(\gamma)$ of the received $\mathrm{SNR}$ at a user is expressed as

$$
f_{r}(\gamma)=\frac{1}{\rho \mu_{r}} e^{-\frac{\gamma}{\rho \mu_{r}}}
$$

where $\rho=P / N_{0}$ denotes the SNR at the transmitter (BS). In general, the RSs are located closer to the MSs than the BS, and, thus, the average SNR of the relay scheme is, in average, greater than that of the direct scheme. However, the relay scheme only has one-half of the degrees of freedom (DOF).

\section{Selection Scheme}

Since a BS has two downlink paths, a BS-MS direct link and a BS-RS-MS relay link, the BS can achieve a larger capacity when it selects a better link among the two communication links. In the selection scheme, we assume that the channel coefficient $h_{d}$ and $h_{r}$ are known to the BS through the feedback from each user. At every transmission, the BS checks which link is better in terms of the achievable capacity and chooses the better link to communicate with the MS, as shown in Fig. 2(c). In this paper, since we assume the channel is independent in every transmission, the channel information should be fed back to the BS at every transmission. However, in practice, the channel information is fed back to the BS with a feedback period shorter than its coherence time. If the direct link is better, the entire transmission time is assigned to the direct transmission between the BS and the MS, while if the relay link is better, then the transmission time is divided into two phases as in the relay scheme. In this scheme, the received signal is given by

(If the direct link is better)

$$
y_{i}=h_{d} s_{i}+n_{i},
$$

(If the relay link is better)

$$
\begin{array}{ll}
y_{i, 1}=n_{i, 1}, & \text { in Phase I, } \\
y_{i, 2}=h_{r} s_{i}+n_{i, 2}, & \text { in Phase II. }
\end{array}
$$

The BS determines the better path based on the achievable capacities of two paths. If the relay scheme which has only one-half degrees of freedom achieves greater capacity, then the BS chooses the relay scheme, otherwise it selects the direct scheme. The instantaneous capacities of the direct path and the relay path are given by $\log _{2}\left(1+\left|h_{d}\right|^{2} P / N_{0}\right)$ and $\frac{1}{2} \log _{2}\left(1+\left|h_{r}\right|^{2} P / N_{0}\right)$, respectively. Therefore, the MS can achieve $\log _{2}\left(\delta_{s}\right)$ where

$$
\delta_{s} \triangleq \max \left(1+\left|h_{d}\right|^{2} P / N_{0}, \sqrt{1+\left|h_{r}\right|^{2} P / N_{0}}\right)
$$

and the PDF of $\delta_{s}$ is given by

$$
\begin{aligned}
& f_{\delta_{s}}(\delta)= \\
& \frac{1}{\rho \mu_{d}} e^{-\frac{\delta-1}{\rho \mu_{d}}}+\frac{2 \delta}{\rho \mu_{r}} e^{-\frac{\delta^{2}-1}{\rho \mu_{r}}}-\left(\frac{1}{\rho \mu_{d}}+\frac{2 \delta}{\rho \mu_{r}}\right) e^{-\frac{\delta-1}{\rho \mu_{d}}-\frac{\delta^{2}-1}{\rho \mu_{r}}}(7)
\end{aligned}
$$


and the detailed derivation of (7) is given in Appendix I.

\section{Cooperative Scheme}

Fig. 2(d) shows the cooperative scheme. The cooperative scheme achieves cooperative diversity by operating the RS as a cooperative transmitter for the BS. It also has two phases: Phase-I for receiving data from the BS at the RS and MS, and Phase-II for sending the data from the RS to the MS. Different from the previous relay scheme, the MS can receive data directly from the BS during Phase-I. Among many cooperative relaying schemes, we choose a repetition scheme which simply transmits the received signal at the relay station. While other cooperative schemes such as distributed space-time-coded protocols [20], [21] require very complex network cooperation among transmitters and receivers, a repetition scheme guarantees the low complexity and ease of implementation. Thus, in this paper, we consider the repetition scheme proposed in [5].

$$
\begin{aligned}
& y_{i, 1}=h_{d} s_{i}+n_{i, 1}, \quad \text { in Phase I, } \\
& y_{i, 2}=h_{r} s_{i}+n_{i, 2}, \quad \text { in Phase II. }
\end{aligned}
$$

The channel coefficients $h_{d}$ and $h_{r}$ are assumed to be known to the MS so that the MS performs maximum ratio combining (MRC). The MRC received signal is given by $y_{i}=$ $\left(\left|h_{d}\right|^{2}+\left|h_{r}\right|^{2}\right) s_{i}+\left(h_{d}^{*} n_{i, 1}+h_{r}^{*} n_{i, 2}\right)$ so that the received SNR for the selection scheme is given by $\delta_{s} \triangleq\left(\left|h_{d}\right|^{2}+\left|h_{r}\right|^{2}\right) P / N_{0}$. The sum of exponential random variables with different mean values, and its PDF is represented as

$$
f_{c}(\gamma)=\frac{e^{-\frac{\gamma}{\rho \mu_{d}}}-e^{-\frac{\gamma}{\rho \mu_{r}}}}{\rho\left(\mu_{d}-\mu_{r}\right)}, \quad\left(\mu_{d} \neq \mu_{r}\right)
$$

and the detailed derivation is given in Appendix II.

\section{PERFORMANCE ANALYSIS OF DOWNLINK CAPACITY}

We first analyze the performance of a single link for each scheme described in Section II. In this section, we analyze the ergodic capacity and outage capacity of each scheme.

\section{A. Ergodic Capacity}

Under the assumption that the channel is stationary and ergodic, we can obtain the ergodic capacity of a single link for each scheme. Instantaneous capacity of a channel with received SNR of the channel, $\gamma$, is defined as $C=\log _{2}(1+\gamma)$. Ergodic capacity is also define as $\mathbf{E}[C]=\mathbf{E}\left[\log _{2}(1+\gamma)\right]$. In the direct scheme, the ergodic capacity is obtained as:

$$
\begin{aligned}
\mathbf{E}\left[C_{d}\right] & =\int_{0}^{\infty} \log _{2}(1+\gamma) f_{d}(\gamma) d \gamma \\
& =-\log _{2}(e) \exp \left(\frac{1}{\rho \mu_{d}}\right) \operatorname{Ei}\left(-\frac{1}{\rho \mu_{d}}\right)
\end{aligned}
$$

where the exponential integral function $\mathrm{Ei}$ is defined as $\mathrm{Ei}(x)=$ $-\int_{-x}^{\infty} \frac{\exp (-t)}{t} d t$. The ergodic capacity is obtained by the relation $\int_{0}^{\infty} \exp (-\mu x) \ln (1+\beta x) d x=-\frac{1}{\mu} \exp \left(\frac{\mu}{\beta}\right) \operatorname{Ei}\left(-\frac{\mu}{\beta}\right)$ [22].
The ergodic capacity of the relay scheme is similarly obtained in a closed-form by

$$
\begin{aligned}
\mathbf{E}\left[C_{r}\right] & =\int_{0}^{\infty} \frac{1}{2} \log _{2}(1+\gamma) f_{r}(\gamma) d \gamma \\
& =-\frac{1}{2} \log _{2}(e) \exp \left(\frac{1}{\rho \mu_{r}}\right) \operatorname{Ei}\left(-\frac{1}{\rho \mu_{r}}\right)
\end{aligned}
$$

where the term $\left(\frac{1}{2}\right)$ is added at the $\log _{2}$ term because the channel utilization of the system is reduced to one-half.

In the selection scheme, the ergodic capacity for a single link cannot be obtained in a closed-form. Hence, we obtain the upper bound of the ergodic capacity using Jensen's inequality [23]. That is, $\mathbf{E}\left\{\log _{2}(x)\right\} \leq \log _{2}(\mathbf{E}\{x\})$ since $\log _{2}$ function is a concave function.

$$
\begin{aligned}
& \mathbf{E}\left[C_{s}\right]=\int_{1}^{\infty} \log _{2}(\gamma) f_{s}(\gamma) d \gamma \\
& \leq \log _{2}\left(\mathbf{E}\left\{\gamma_{s}\right\}\right) \\
& =\log _{2}\left\{1+\rho \mu_{d}+\frac{1}{2} \sqrt{\pi \rho \mu_{r}} \mathrm{e}^{\frac{1}{\rho \mu_{r}}}\left[1-\operatorname{erf}\left(\sqrt{\frac{1}{\rho \mu_{r}}}\right)\right]\right. \\
& \left.\quad-\frac{1}{2} \sqrt{\pi \rho \mu_{r}} \mathrm{e}^{\frac{1}{4} \frac{\left(2 \rho \mu_{d}+\rho \mu_{r}\right)^{2}}{\rho^{3} \mu_{d}^{2} \mu_{r}}}\left[1-\operatorname{erf}\left(\frac{1}{2} \frac{2 \rho \mu_{d}+\rho \mu_{r}}{\rho \mu_{d} \sqrt{\rho \mu_{r}}}\right)\right]\right\}
\end{aligned}
$$

where the error function is defined as erf $(x)=\frac{2}{\sqrt{\pi}} \int_{0}^{x} \exp \left(-t^{2}\right)$ $d t$ [22].

In the cooperative scheme, a closed-form ergodic capacity is obtained as

$$
\begin{aligned}
\mathbf{E}\left[C_{c}\right]=\int_{0}^{\infty} \frac{1}{2} \log _{2}(1+\gamma) f_{c}(\gamma) d \gamma \\
=\frac{\log _{2}(e) \mu_{r} \mathrm{e}^{\frac{1}{\rho \mu_{r}}} \mathrm{Ei}\left(-\frac{1}{\rho \mu_{r}}\right)-\mu_{d} \mathrm{e}^{\frac{1}{\rho \mu_{d}}} \operatorname{Ei}\left(-\frac{1}{\rho \mu_{d}}\right)}{2\left(\mu_{d}-\mu_{r}\right)}
\end{aligned}
$$

Before we analyze the result of ergodic capacities, we define the average channel gain difference in $\mathrm{dB}$ between $\mu_{r}$ and $\mu_{d}$ as $\mu_{\mathrm{diff}}(\mathrm{dB}) \triangleq \mu_{r}(\mathrm{~dB})-\mu_{d}(\mathrm{~dB})$. When $\mu_{\text {diff }}$ is large, the RS-MS channel is better than the BS-MS channel for an MS. For example, if $\mu_{\text {diff }}$ is set to $3 \mathrm{~dB}$, it represents that the RS-MS channel is two times better in average SNR than the BS-MS channel. Fig. 3 shows the result of the ergodic capacities for four different schemes when $\mu_{d}$ is set to $0 \mathrm{~dB}$. The result shows the ergodic capacity for varying $\mu_{\text {diff }}$ values. All the results are obtained from the closed-form expressions of ergodic capacities, except that the ergodic capacity of the selection scheme is generated from Monte Carlo simulation. From the figure, we can observe that the selection scheme outperforms all the other schemes in the range of $\mu_{\text {diff }}$ from $0 \mathrm{~dB}$ to $15 \mathrm{~dB}$. In the range of $\mu_{\text {diff }}>15$, the cooperative scheme outperforms the selection scheme. The capacity of the relay scheme is always below the capacities of the selection and the cooperative schemes, and the capacity of the direct scheme remains the same. For more discussions on the performance comparisons, we compare the instantaneous Shan- 


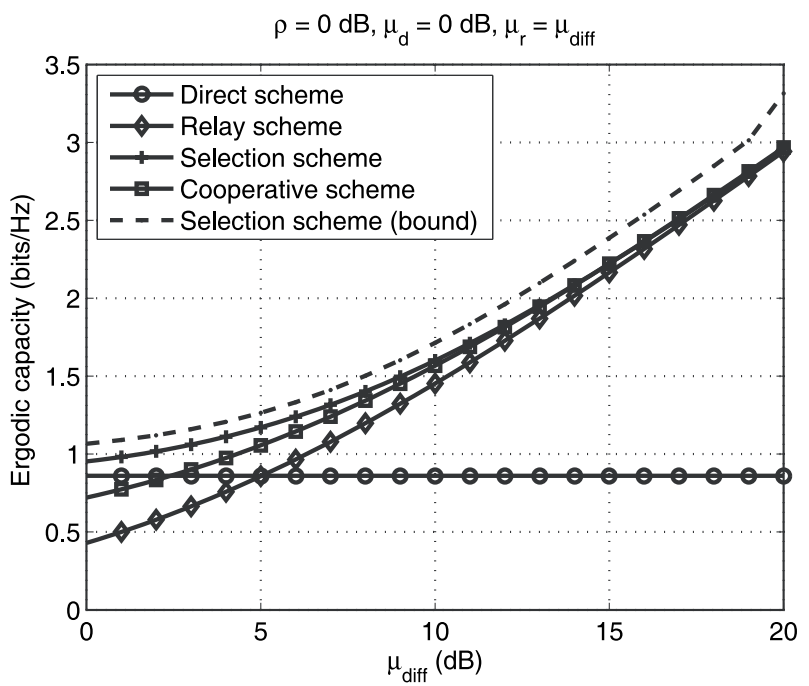

Fig. 3. Link ergodic capacities for four different schemes.

non capacity when the instant channel realization is given.

$$
\begin{aligned}
C_{d} & =\log _{2}\left(1+\rho\left|h_{d}\right|^{2}\right), \\
C_{r} & =\frac{1}{2} \log _{2}\left(1+\rho\left|h_{r}\right|^{2}\right), \\
C_{s} & =\max \left\{\log _{2}\left(1+\rho\left|h_{d}\right|^{2}\right), \frac{1}{2} \log _{2}\left(1+\rho\left|h_{r}\right|^{2}\right)\right\}, \\
C_{c} & =\frac{1}{2} \log _{2}\left(1+\rho\left(\left|h_{d}\right|^{2}+\left|h_{r}\right|^{2}\right)\right) .
\end{aligned}
$$

From these equations, we can observe that $C_{s}$ is always greater than $C_{d}$ and $C_{r}$. Furthermore, $C_{c}$ is always greater than $C_{r}$. If the RS-MS channel is far better than the BS-MS channel, i.e., $\mu_{\text {diff }} \rightarrow \infty, C_{s}$ is similar to $C_{r}$ since the BS almost surely chooses the relaying path all the time. In this high $\mu_{\text {diff }}$ case, the order of the capacities of the four schemes is $C_{c}>C_{r}>$ $C_{s}>C_{d}$. In Fig 3, however, in the setting where $\mu_{d}=0 \mathrm{~dB}$ and $0 \mathrm{~dB}<\mu_{\mathrm{diff}}<15 \mathrm{~dB}$, the selection scheme yields the best performance due to the fact that it can obtain transmission diversity by selecting a better channel between the BS-MS channel and the RS-MS channel. When $\mu_{\text {diff }}$ is approximately $15(\mathrm{~dB})$ (i.e., when the RS-MS channel gain is 32 times greater than the BS-MS channel gain), there is a cross point where the cooperative scheme outperforms the selection scheme. The relay scheme and the cooperative scheme outperform the direct scheme when the SNR difference is larger than $5 \mathrm{~dB}$ and $2 \mathrm{~dB}$, respectively. Therefore, when there is a sufficient number of RSs in a cell so that RS-MS channels are generally at least two times better than BS-MS channels, both the selection scheme and the cooperative scheme perform well due to their transmission diversity. In addition, the cooperative scheme always yields a better capacity than the relay scheme because each MS receives the data in Phases I and II in the cooperative scheme, while each MS receives the data only in Phase II in the relay scheme.

\section{B. Outage Capacity}

In this subsection, we obtain the outage capacity for four different schemes. Outage capacity is here defined as $C_{\epsilon} \triangleq$

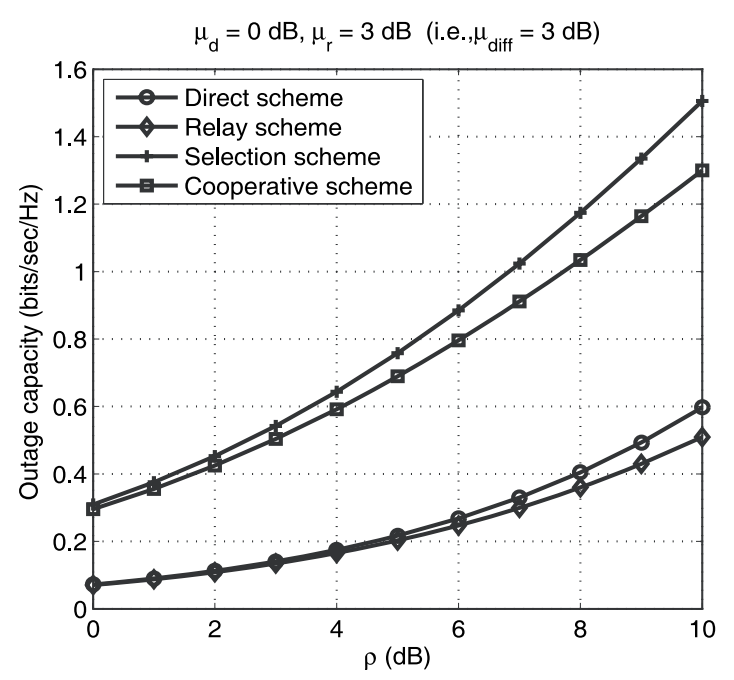

Fig. 4. Outage capacities for four different schemes for varying $\rho$.

$\log _{2}\left(1+G^{-1}(1-\epsilon)\right)$, where $G$ is the complementary cumulative distribution function (CDF) of $|h|^{2}$, i.e., $G(x) \triangleq$ $P\left\{|h|^{2}>x\right\}$.

The function $G$ for the direct scheme is given by

$$
G_{d}^{-1}(x)=-\ln (x) \rho \mu_{d}
$$

and for the relay scheme,

$$
G_{r}^{-1}(x)=-\ln (x) \rho \mu_{r} .
$$

For the selection and cooperative schemes, since the inverse function of the complementary CDF does not exist, we numerically obtain $G_{s}^{-1}(x)$ and $G_{c}^{-1}(x)$.

Fig. 4 shows the result of the outage capacities with a reasonable error probability of $\epsilon=0.05$ in typical cellular systems, for the four different schemes. The result shows the relationship between the outage capacity for the four schemes and input $\rho=P / N_{0}$. The result is obtained under the condition that $\mu_{\text {diff }}$ is set to $3 \mathrm{~dB}$, which means that the RS-MS channel is two times better in terms of average SNR than the BS-MS channel due to the proximity of the RS or the large transmit power at the RS. The selection scheme outperforms the other three schemes in terms of outage capacity. The outage capacities of the selection and cooperative schemes are much better than those of the direct and relay schemes because the first two schemes exploiting the transmit diversity yield low outage performance. For a low SNR region, the cooperative scheme has almost the same outage capacity as the selection scheme.

\section{SYSTEM PERFORMANCE ANALYSIS WITH MULTIUSER DIVERSITY}

In this section, we analyze the system performance with multiple links for four different schemes. In the system performance analysis, we assume that there exist $N$ MSs in a cell and all MSs communicate with one BS at the cell center. In practical cellular systems, since the location of the $i$ th MS is uniformly distributed over a cell area, it has independent $\mu_{d, i}$ and $\mu_{r, i}$ depending on its distances to the serving BS. However, for this 
realistic model, it is hard to mathematically analyze the system performance. Besides, the effect of relaying schemes on multiuser diversity is softened by the effect of the location of MSs. Thus, in order to investigate the effect of relaying schemes on multiuser diversity, we assume that all $N$ MS-MS channels are i.i.d. Rayleigh channels with an average channel gain of $\mu_{d}$, and all $N$ RS-MS channels are i.i.d. Rayleigh channels with an average channel gain $\mu_{r}$. In this multiuser system, a maximum SNR scheduling scheme is used. The maximum SNR scheduling scheme is optimal for achieving the maximum multiuser diversity gain, but fairness and delay problems remain. In many practical next-generation mobile communication systems, the proportional fairness (PF) scheduling scheme is widely used to achieve multiuser diversity gain because it guarantees the fairness of the systems. In this paper, we consider the maximum SNR scheduling scheme for mathematical simplicity.

We estimate the ergodic capacity of a system with $N$ users using the expression $C(N)=\mathbf{E}\left\{\log _{2}\left(1+\gamma_{\max }\right)\right\}$, where $\gamma_{\max }=\max \left\{\gamma_{1}, \gamma_{2}, \cdots, \gamma_{N}\right\}$ and $\gamma_{i}$ represents the SNR of the $i$-th user, and $\gamma_{\max }$ represents the SNR of the user whose instantaneous channel gain is the largest within the system. From order statistics, we obtain the CDF of $\gamma_{\max }, F_{\max }(\gamma)=$ $\{F(\gamma)\}^{N}$ given that all $\gamma_{i}$ 's are i.i.d. and have their individual cumulative distribution function (CDF), $F(\gamma)$. From $F_{\max }(\gamma)$, we can also obtain the PDF of $\gamma_{\max }, f_{\max }(\gamma)=$ $N[F(\gamma)]^{N-1} f(\gamma)$.

In the direct scheme, the multiuser ergodic capacity is obtained in a closed-form,

$$
\begin{aligned}
\mathbf{E} & {\left[C_{d}(N)\right] } \\
& =\int_{0}^{\infty} \log _{2}(1+\gamma) f_{d, \max }(\gamma) d \gamma \\
= & -N \log _{2}(e) \cdot \\
& \sum_{k=0}^{N-1}\left\{\left(\begin{array}{c}
N-1 \\
k
\end{array}\right)(-1)^{k} \frac{1}{k+1} e^{\frac{k+1}{\rho \mu_{d}}} \operatorname{Ei}\left(-\frac{k+1}{\rho \mu_{d}}\right)\right\}
\end{aligned}
$$

where $f_{d, \max }(\gamma)=N\left[F_{d}(\gamma)\right]^{N-1} f_{d}(\gamma)$ and $f(\gamma)$ is given in (2). The detailed derivation of (20) is given in Appendix III.

Similarly, in the relay scheme, the multiuser ergodic capacity is obtained in a closed-form,

$$
\begin{aligned}
\mathbf{E}[ & \left.C_{r}(N)\right] \\
= & \int_{0}^{\infty} \frac{1}{2} \log _{2}(1+\gamma) f_{r, \max }(\gamma) d \gamma \\
= & -\frac{N \log _{2}(e)}{2} . \\
& \sum_{k=0}^{N-1}\left\{\left(\begin{array}{c}
N-1 \\
k
\end{array}\right)(-1)^{k} \frac{1}{k+1} e^{\frac{k+1}{\rho \mu_{r}}} \operatorname{Ei}\left(-\frac{k+1}{\rho \mu_{r}}\right)\right\}
\end{aligned}
$$

where $f_{r, \max }(\gamma)=N\left[F_{r}(\gamma)\right]^{N-1} f_{r}(\gamma)$ and $f_{r}(\gamma)$ is given in (4).

In the selection scheme, there is no closed-form solution for the multiuser ergodic capacity. Thus, we obtain an upper bound which can be applied to any set of i.i.d. random variables. The upper bound was found in [23]:

$$
\begin{aligned}
\mathbf{E}\left[C_{s}(N)\right] & =\int_{1}^{\infty} \log _{2}(\delta) f_{s, \max }(\delta) d \delta \\
& \leq \log _{2}\left(\mu_{s}+\frac{N-1}{\sqrt{2 N-1}} \sigma_{s}\right)
\end{aligned}
$$

where $f_{s, \max }(\delta)=N\left[F_{s}(\delta)\right]^{N-1} f_{s}(\delta)$ and $f_{s}(\delta)$ is given in (7). The terms $\mu_{s}$ and $\sigma_{s}$ denote the mean and standard deviation of $\delta_{s}$ and are expressed as

$$
\begin{aligned}
\mu_{s}=1 & +\mu_{d}+\frac{1}{2} \sqrt{\pi \mu_{r}} \exp \left(\frac{1}{\mu_{r}}\right)\left(1-\operatorname{erf}\left(\sqrt{\frac{1}{\mu_{r}}}\right)\right) \\
& -\frac{1}{2} \sqrt{\pi \mu_{r}} \exp \left(\frac{1}{4} \frac{\left(2 \mu_{d}+\mu_{r}\right)^{2}}{\mu_{d}^{2} \mu_{r}}\right) . \\
& \left\{1-\operatorname{erf}\left(\frac{1}{2} \frac{\left(2 \mu_{d}+\mu_{r}\right)}{\mu_{d} \sqrt{\mu_{r}}}\right)\right\}, \\
\sigma_{s}^{2}=2 & \left(1+\mu_{d}+\mu_{d}^{2}\right)+\mu_{r} \\
& +\frac{\sqrt{\mu_{r}}}{2 \mu_{d}}\left[\mu_{r} \sqrt{\pi} \exp \left(\frac{1}{4} \frac{\left(2 \mu_{d}+\mu_{r}\right)^{2}}{\mu_{d}^{2} \mu_{r}}\right) .\right. \\
& \left\{1-\operatorname{erf}\left(\frac{1}{2} \frac{\left(2 \mu_{d}+\mu_{r}\right)}{\mu_{d} \sqrt{\mu_{r}}}\right)\right\} \\
& \left.-2 \sqrt{\mu_{r}} \mu_{d}-\frac{2 \mu_{d}}{\sqrt{\mu_{r}}}\right]-\mu_{s}^{2} .
\end{aligned}
$$

Finally, in the cooperative scheme, a closed-form solution for the multiuser ergodic capacity is obtained as follows:

$$
\begin{aligned}
\mathbf{E}\left[C_{c}(N)\right] & \int_{0}^{\infty} \frac{1}{2} \log _{2}(1+\gamma) f_{c, \max }(\gamma) d \gamma \\
= & \frac{N \log _{2}(e)}{2 \rho\left(\mu_{d}-\mu_{r}\right)} \sum_{k=0}^{N-1}\left[\left(\begin{array}{c}
N-1 \\
k
\end{array}\right)\left(\frac{1}{\mu_{r}-\mu_{d}}\right)^{k} .\right. \\
& \sum_{i=0}^{k}\left\{( - 1 ) ^ { i } \mu _ { r } ^ { i } \mu _ { d } ^ { k - i } \left(\mathbf{I}\left(\frac{i+1}{\rho \mu_{r}}+\frac{k-i}{\rho \mu_{d}}\right)\right.\right. \\
& \left.\left.\left.-\mathbf{I}\left(\frac{i}{\rho \mu_{r}}+\frac{k-i+1}{\rho \mu_{d}}\right)\right)\right\}\right] \\
\mathbf{I}(a) \triangleq & \int_{0}^{\infty} \ln (1+\gamma) \mathrm{e}^{-a \gamma} d \gamma=-\frac{1}{a} \mathrm{e}^{a} \operatorname{Ei}(-a) .
\end{aligned}
$$

The detailed procedure of the mathematical derivation is given in Appendix IV.

Figs. 5 and 6 show the ergodic capacities for the four different schemes with $N$ users in a cell when $\mu_{\text {diff }}$ is set to $3 \mathrm{~dB}$ and $9 \mathrm{~dB}$, respectively. From the two figures, we can find the effect of multiuser diversity and selection diversity. In Fig. 5, the selection scheme yields the best performance over all ranges and the selection diversity improves the performance of the selection scheme by approximately $20 \%$, compared with that of the direct scheme in the case of $N=1$. As the number of users increases, both the selection and direct schemes exploit multiuser diversity. In our analysis, the ergodic capacities of the two schemes are nearly identical as the number of users increases. From this 


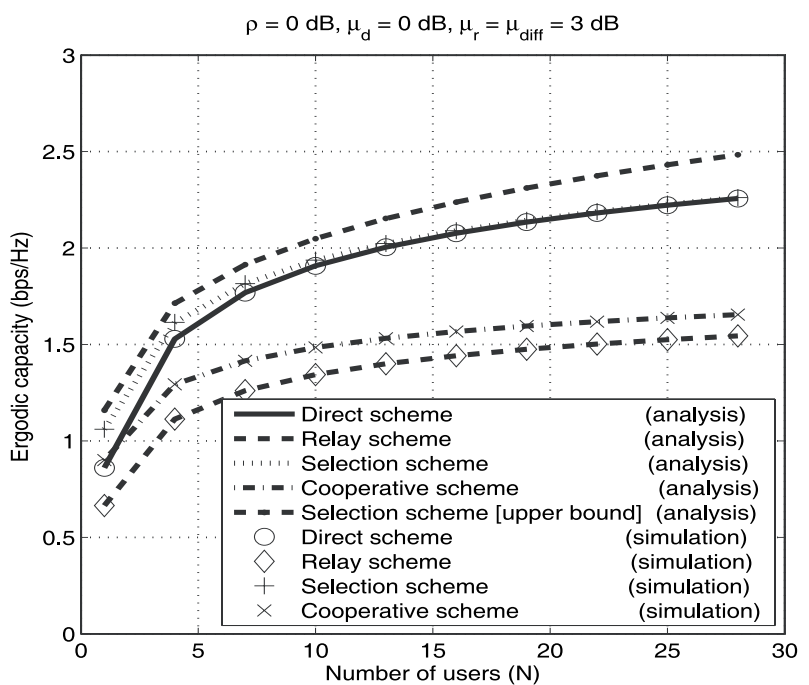

Fig. 5. System capacities for four different schemes for varying $N$ values when $\mu_{\text {diff }}$ is set to $3 \mathrm{~dB}$.

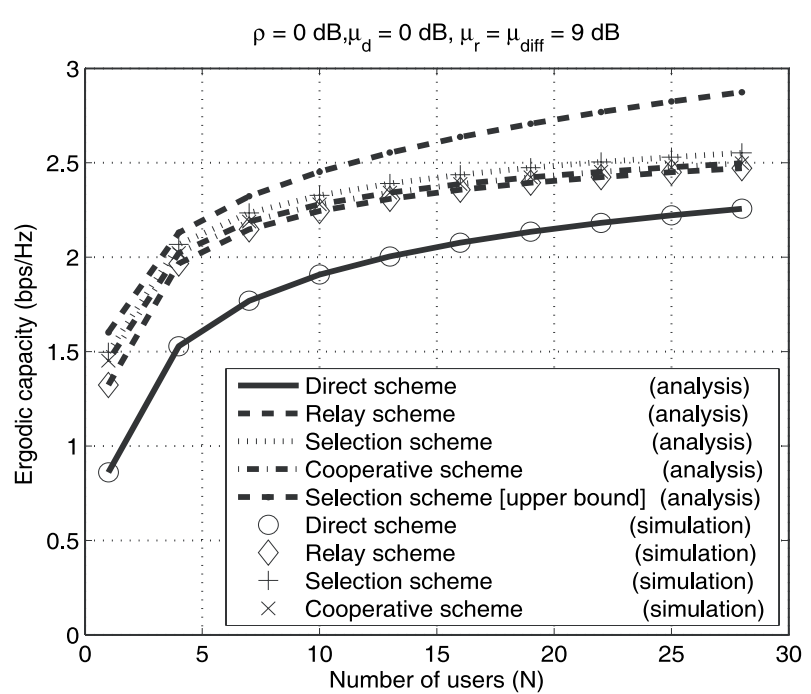

Fig. 6. System capacities for four different schemes for varying $N$ values when $\mu_{\text {diff }}$ is set to $9 \mathrm{~dB}$.

result, we observe that the multiuser diversity in the selection scheme is more dominant than its own selection diversity because the system has no big difference in the performance, compared to the direct scheme which only exploits the multiuser diversity. Therefore, for a system with low $\mu_{\text {diff }}$ values, the direct scheme achieves the best multiuser ergodic capacity when $N$ is more than 10 . In addition, since the direct scheme does not require any $\mathrm{RS}$ and high scheduling complexity, it has some advantages over other complex schemes.

On the other hand, in Fig. 6, for a given system with $\mu_{\text {diff }}=$ $9 \mathrm{~dB}$, since the RS-MS channel is much better than the BSMS channel, the selection scheme yields the best performance. In this case, the relay scheme achieves almost the best performance as described in the previous case. Multiuser diversity in the selection scheme is dominant and the direct scheme also exploits multiuser diversity well, and, thus, the performance of both schemes is similar for a large number of users.

The selection scheme outperforms all the other schemes in a multiuser environment, while the direct scheme and the relay scheme almost achieve the performance of the selection scheme due to dominant multiuser diversity at low $\mu_{\text {diff }}$ values and high $\mu_{\text {diff }}$ values, respectively.

\section{A. Analysis of the Effect of Cooperative and Selection Diversi- ties on Multiuser Diversity}

We observed how the four different relaying schemes perform in a multiuser environment. In this subsection, we analyze the effect of cooperative and selection diversity techniques on multiuser diversity in a downlink cellular system with relays. We compare the characteristics of the distributions of onelink capacities for the four different relaying schemes. Eqs. (27) through (30) represent the PDFs of the one-link instantaneous capacities for the direct, the relay, the selection, and the cooperative schemes, respectively. The following four PDFs are obtained by converting the PDFs of the SNRs for the four schemes, given in (2), (4), (7), and (9), into the PDFs of the capacities.

$$
\begin{aligned}
f_{D, C_{1}}(c)= & \frac{\ln (2)}{\rho \mu_{d}} \exp \left(-\frac{2^{c}-1}{\rho \mu_{d}}\right) 2^{c}, \\
f_{R, C_{1}}(c)= & \frac{\ln (2)}{\rho \mu_{r}} \exp \left(-\frac{4^{c}-1}{\rho \mu_{r}}\right) 4^{c} 2, \\
f_{S, C_{1}}(c)= & \ln (2) 2^{c}\left[\frac{1}{\rho \mu_{d}} \exp \left(-\frac{2^{c}-1}{\rho \mu_{d}}\right)+\right. \\
& \frac{2^{c+1}}{\rho \mu_{r}} \exp \left(-\frac{4^{c}-1}{\rho \mu_{r}}\right) \\
- & \left.\left(\frac{1}{\rho \mu_{d}}+\frac{2^{c+1}}{\rho \mu_{r}}\right) \exp \left(-\frac{2^{c}-1}{\rho \mu_{d}}-\frac{4^{c}-1}{\rho \mu_{r}}\right)\right], \\
f_{C, C_{1}}(c)= & \frac{\ln (2)}{\rho\left(\mu_{d}-\mu_{r}\right)} 4^{c} 2 \cdot \\
& \left(\exp \left(-\frac{2^{c}-1}{\rho \mu_{d}}\right)-\exp \left(-\frac{4^{c}-1}{\rho \mu_{r}}\right)\right)
\end{aligned}
$$

where $c$ denotes the one-link instantaneous capacity.

From the PDFs, we can obtain the CDFs of the capacities as (31) through (34).

$$
\begin{aligned}
F_{D, C_{1}}(c)= & 1-\exp \left(-\frac{2^{c}-1}{\rho \mu_{d}}\right) \\
F_{R, C_{1}}(c)= & 1-\exp \left(-\frac{4^{c}-1}{\rho \mu_{r}}\right) \\
F_{S, C_{1}}(c)= & 1-\exp \left(-\frac{2^{c}-1}{\rho \mu_{d}}\right)-\exp \left(-\frac{4^{c}-1}{\rho \mu_{r}}\right)+ \\
& \quad \exp \left(-\frac{4^{c} \mu_{d}-\mu_{d}+2^{c} \mu_{r}-\mu_{r}}{\rho \mu_{d} \mu_{r}}\right) \\
F_{C, C_{1}}(c)= & 1-\frac{\exp \left(-\frac{4^{c}-1}{\rho \mu_{d}}\right) \mu_{d}-\exp \left(-\frac{4^{c}-1}{\rho \mu_{r}}\right) \mu_{r}}{\mu_{d}-\mu_{r}}
\end{aligned}
$$

Now, we analyze the distributions of multiuser system capacities for four different schemes. Since we adopt the opportunistic scheduling which instantaneously chooses the MS with the largest SNR value, the multiuser system capacity is defined as,

$$
C(N)=\log _{2}\left(1+\max _{1 \leq i \leq N} \gamma_{i}\right)=\max _{1 \leq i \leq N}\left\{C_{1, i}\right\}
$$




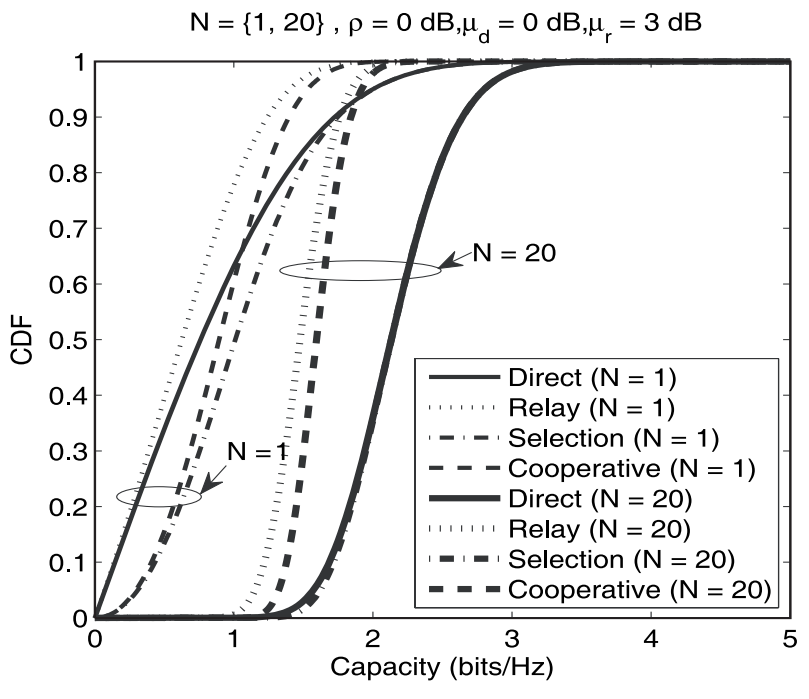

Fig. 7. CDFs of capacities for four different relaying schemes when $N=\{1,20\}, \rho=0 \mathrm{~dB}, \mu_{d}=0 \mathrm{~dB}$, and $\mu_{r}=3 \mathrm{~dB}$.

Table 1. Means and variances of capacities for four different relaying schemes when $N=1$.

\begin{tabular}{|c||c|c|}
\hline Relaying scheme & $\mathbf{E}\left[C_{1}\right]$ & $\operatorname{Var}\left[C_{1}\right]$ \\
\hline \hline Direct & 0.86 & 0.36 \\
\hline Relay & 0.66 & 0.17 \\
\hline Selection & 1.06 & 0.26 \\
\hline Cooperative & 0.90 & 0.14 \\
\hline
\end{tabular}

where we define $C_{1, i}=\log _{2}\left(1+\gamma_{i}\right)$. From order statistics, $\mathrm{CDF}$ of $C(N)$ is expressed as

$$
F_{C_{N}}=\prod_{i=1}^{N} F_{C_{i}}=\left\{F_{C_{1}}\right\}^{N}
$$

because all the $C_{1, i}$ 's are independent for $1 \leq i \leq N$. Therefore, we obtain the CDFs for four different schemes in multiuser environment using (31), (32), (33), and (34), and the relation noted in (36). Fig. 7 shows the CDFs of capacities for the four different relaying schemes when $N$ is set to 1 and 20. We also set the parameter values of a typical cellular system: $\rho=0 \mathrm{~dB}$, $\mu_{d}=0 \mathrm{~dB}$, and $\mu_{r}=3 \mathrm{~dB}$. We first observe $N=1$ case. The selection scheme outperforms all the other schemes in the CDF analysis as we have observed in Section IV. Both the direct scheme and the relay scheme have relatively lower capacity than the selection and the cooperative schemes because the former two schemes do not have any diversity techniques, while the latter two schemes achieve the cooperative and selection diversities, respectively, so that the probability of very low channel gains is dramatically decreased.

Table 1 lists the mean and the variance values for the four schemes when $N=1$. The mean values of the direct and the relay schemes are lower than those of the selection and the cooperative schemes. Note that in Table 1, the variance of the direct scheme is relatively higher than that of other schemes. The large variance of the direct scheme can be also observed in Fig. 7. As shown in Fig. 7, the CDF of the direct scheme has relatively a large variance among the four schemes. The CDF curve of the
Table 2. Means and variances of capacities for four different relaying schemes when $N=20$.

\begin{tabular}{|c||c|c|}
\hline Relaying scheme & $\mathbf{E}\left[C_{20}\right]$ & $\operatorname{Var}\left[C_{20}\right]$ \\
\hline \hline Direct & 2.15 & 0.14 \\
\hline Relay & 1.48 & 0.04 \\
\hline Selection & 2.16 & 0.13 \\
\hline Cooperative & 1.60 & 0.03 \\
\hline
\end{tabular}

direct scheme has a very long-tail distribution, almost identical to the CDF curve of the selection scheme. The impact of this very large variance of the direct scheme will be analyzed later in this section.

When $N$ is set to 20 , the order in mean values among the four schemes is not the same as that in the single MS case. Interestingly, in the multiuser environment, the performance of the direct scheme almost follows that of the selection scheme and their performance outperforms the other schemes. The cooperative scheme, which yields better mean performance than the direct scheme in the single MS case, shows low performance as the relay scheme. This phenomenon can be characterized by analyzing the variances of capacities of the four different schemes. Table 2 shows the means and variances for the four schemes when $N=20$. First, we can observe that the means of all schemes increase, while the variances decrease through opportunistic scheduling. An opportunistic scheduling gain is obtained from the fluctuation of the fading channels, in other words, a multiuser diversity gain is achieved from the variance of the channel. Thus, the opportunistic scheduling increases the mean and decreases the variance. Without the opportunistic scheduling, the direct scheme has the highest variance among the four schemes, and, thus, the multiuser diversity gain through the opportunistic scheduling is greatly achieved in the direct scheme. Even though the mean capacity of the direct scheme is lower than that of the cooperative scheme without the opportunistic scheduling, the direct scheme performs better than the cooperative scheme with the opportunistic scheduling in the mean capacity sense.

For asymptotical analysis, we adopt the bound for the first moment of order statistics. If all $X_{i}$ 's (for $i=1, \cdots, N$ ) are independent identically distributed (i.i.d.) random variables, the mean of the largest value among $X_{i}$ 's is given by [23]

$$
\mathbf{E}\left[\max _{1 \leq i \leq N} X_{i}\right] \leq \mathbf{E}\left[X_{1}\right]+\frac{N-1}{\sqrt{2 N-1}} \sqrt{\operatorname{Var}\left[X_{1}\right]}
$$

As $N$ goes to infinity, the left-hand-side term is limited only by the variance of $X_{1}$ and $N$. In our study, we can take the random variable $X_{1}$ as the instantaneous one-link capacity of a user, $C_{1}$. From the bound, we can obtain the intuition as follows: as the number of MSs in the cell, $N$, goes to infinity, the mean of the multiuser system capacity is determined only by the variance of the one-link capacity. This is why the multiuser system mean capacity of the direct scheme, whose variance is the largest among four schemes, drastically increases through the opportunistic scheduling. 


\section{CONCLUSIONS}

We mathematically analyze the ergodic and outage capacities in downlink for four different data relaying schemes: A direct scheme, a relay scheme, a selection scheme, and a cooperative scheme. Furthermore, we derive closed-form solutions or upper bound of the ergodic capacity for the schemes. The analytical results agree well with computer simulation results. The results show that the selection scheme outperforms the other three schemes in terms of link ergodic capacity, outage capacity, and system ergodic capacity. We also investigate the effect of the cooperative and selection diversity techniques on multiuser diversity. The result shows that the cooperative and selection diversity techniques in cellular systems with relays limit the multiuser diversity gain that could have been achieved by the opportunistic scheduling.

\section{APPENDICES}

\section{THE PDF OF $\delta_{s}$ IN THE DERIVATION OF THE CAPACITY OF SELECTION SCHEME}

We let new random variables $X \triangleq 1+\left|h_{d}\right|^{2} P / N_{0}$ and $Y \triangleq$ $\sqrt{1+\left|h_{r}\right|^{2} P / N_{0}}$. Since we assume the channels are Rayleigh distributed, we obtain

$$
\begin{aligned}
& f_{X}(x)=\frac{1}{\rho \mu_{d}} \exp \left(-\frac{x-1}{\rho \mu_{d}}\right), \\
& f_{Y}(y)=\frac{2 y}{\rho \mu_{r}} \exp \left(-\frac{y^{2}-1}{\rho \mu_{r}}\right) .
\end{aligned}
$$

Since $\delta_{s}=\max (X, Y)$ from (6), from order statistics we obtain the CDF of $\delta_{s}$ given by

$$
\begin{aligned}
F_{\delta_{s}}(\delta)= & F_{X}(x) F_{Y}(y) \\
= & 1-\exp \left(-\frac{x-1}{\rho \mu_{d}}\right)-\exp \left(-\frac{y^{2}-1}{\rho \mu_{r}}\right) \\
& +\exp \left(-\left(\frac{x-1}{\rho \mu_{d}}+\frac{y^{2}-1}{\rho \mu_{r}}\right)\right) .
\end{aligned}
$$

By differentiating it, we finally obtain (7).

\section{THE DISTRIBUTION OF THE SUM OF TWO EXPONENTIAL RANDOM VARIABLES}

We compute the convolution needed in the derivation of the distribution.

$$
\begin{aligned}
\exp & (-a x) * \exp (-b x) \\
= & \int_{0}^{x} \exp (-a(x-u)) \exp (-b u) \mathrm{d} u \\
= & \exp (-a x) \frac{\exp ((a-b) x)-1}{a-b} \\
= & \frac{\exp (-b x)-\exp (-a x)}{a-b} .
\end{aligned}
$$

For $n=2$, if the two random variables, $X_{1}$ and $X_{2}$, have their parameters $\lambda_{1}$ and $\lambda_{2}$ and they are different [24],

$$
\begin{aligned}
f_{X_{1}+X_{2}}(x) & =f_{X_{1}}(x) * f_{X_{2}}(x) \\
& =\lambda_{1} \lambda_{2} \frac{\exp \left(-\lambda_{2} x\right)-\exp \left(-\lambda_{1} x\right)}{\lambda_{1}-\lambda_{2}} \\
& =\lambda_{1} \lambda_{2}\left[\frac{\exp \left(-\lambda_{1} x\right)}{\lambda_{2}-\lambda_{1}}+\frac{\exp \left(-\lambda_{2} x\right)}{\lambda_{1}-\lambda_{2}}\right]
\end{aligned}
$$

Thus, the PDF of the SNR of the cooperative scheme is given by

$$
f_{c}(\gamma)=\frac{\exp \left(-\frac{\gamma}{\rho \mu_{d}}\right)-\exp \left(-\frac{\gamma}{\rho \mu_{r}}\right)}{\rho\left(\mu_{d}-\mu_{r}\right)}, \quad\left(\mu_{d} \neq \mu_{r}\right) .
$$

\section{MULTIUSER ERGODIC CAPACITY OF THE DIRECT SCHEME}

We provide the detailed derivation of the ergodic capacity of the direct scheme when $N>1$.

$$
\begin{aligned}
& \mathbf{E}\left[C_{d}(N)\right]=\int_{0}^{\infty} \log _{2}(1+\gamma) f_{\max }(\gamma) d \gamma \\
& =\int_{0}^{\infty} \log _{2}(1+\gamma) N\left(1-e^{-\frac{\gamma}{\rho \mu_{d}}}\right)^{N-1} \frac{1}{\rho \mu_{d}} e^{-\frac{\gamma}{\rho \mu_{d}}} d \gamma \\
& =\frac{N}{\rho \mu_{d}} \int_{0}^{\infty} \log _{2}(1+\gamma)\left(1-e^{-\frac{\gamma}{\rho \mu_{d}}}\right)^{N-1} e^{-\frac{\gamma}{\rho \mu_{d}}} d \gamma \\
& =\frac{N \log _{2}(e)}{\rho \mu_{d}} \text {. } \\
& \int_{0}^{\infty} \ln (1+\gamma)\left\{\sum_{k=0}^{N-1}\left(\begin{array}{c}
N-1 \\
k
\end{array}\right)(-1)^{k} e^{-\frac{k \gamma}{\rho \mu_{d}}}\right\} e^{-\frac{\gamma}{\rho \mu_{d}}} d \gamma \\
& =\frac{N \log _{2}(e)}{\rho \mu_{d}} \text {. } \\
& \sum_{k=0}^{N-1}\left\{\left(\begin{array}{c}
N-1 \\
k
\end{array}\right)(-1)^{k} \int_{0}^{\infty} \ln (1+\gamma) e^{-\frac{(k+1) \gamma}{\rho \mu_{d}}} d \gamma\right\} \\
& =\frac{N \log _{2}(e)}{\rho \mu_{d}} \text {. } \\
& \sum_{k=0}^{N-1}\left\{\left(\begin{array}{c}
N-1 \\
k
\end{array}\right)(-1)^{k}\left[-\frac{\rho \mu_{d}}{k+1} e^{\frac{k+1}{\rho \mu_{d}}} \operatorname{Ei}\left(-\frac{k+1}{\rho \mu_{d}}\right)\right]\right\} \\
& =-N \log _{2}(e) \text {. } \\
& \sum_{k=0}^{N-1}\left\{\left(\begin{array}{c}
N-1 \\
k
\end{array}\right)(-1)^{k} \frac{1}{k+1} e^{\frac{k+1}{\rho \mu_{d}}} \mathrm{Ei}\left(-\frac{k+1}{\rho \mu_{d}}\right)\right\} \text {. }
\end{aligned}
$$

\section{MULTIUSER ERGODIC CAPACITY OF THE COOPERATIVE SCHEME}

The derivation of (26) is given in this Appendix.

$$
\mathbf{E}\left[C_{c}(N)\right]=\int_{0}^{\infty} \frac{1}{2} \log _{2}(1+\gamma) f_{c, \max }(\gamma) d \gamma
$$




$$
\begin{aligned}
& =\frac{\log _{2}(e)}{2} \int_{0}^{\infty} \ln (1+\gamma) N \\
& \left(1-\frac{\mu_{d} e^{-\frac{\gamma}{\rho \mu_{d}}}-\mu_{r} e^{-\frac{\gamma}{\rho \mu_{r}}}}{\mu_{d}-\mu_{r}}\right)^{N-1}\left(\frac{e^{-\frac{\gamma}{\rho \mu_{d}}}-e^{-\frac{\gamma}{\rho \mu_{r}}}}{\rho\left(\mu_{d}-\mu_{r}\right)}\right) d \gamma \\
& =\frac{N \log _{2}(e)}{2 \rho\left(\mu_{d}-\mu_{r}\right)} \text {. } \\
& \int_{0}^{\infty} \ln (1+\gamma)\left(e^{-\frac{\gamma}{\rho \mu_{d}}}-e^{-\frac{\gamma}{\rho \mu_{r}}}\right) \text {. } \\
& {\left[\sum_{k=0}^{N-1}\left(\begin{array}{c}
N-1 \\
k
\end{array}\right)(-1)^{k}\left(\frac{\mu_{d} e^{-\frac{\gamma}{\rho \mu_{d}}}-\mu_{r} e^{-\frac{\gamma}{\rho \mu_{r}}}}{\mu_{d}-\mu_{r}}\right)^{k}\right] d \gamma} \\
& =\frac{N \log _{2}(e)}{2 \rho\left(\mu_{d}-\mu_{r}\right)} \int_{0}^{\infty} \ln (1+\gamma)\left(e^{-\frac{\gamma}{\rho \mu_{d}}}-e^{-\frac{\gamma}{\rho \mu_{r}}}\right) \text {. } \\
& {\left[\sum_{k=0}^{N-1}\left(\begin{array}{c}
N-1 \\
k
\end{array}\right)(-1)^{k}\left(\frac{\mu_{d} e^{-\frac{\gamma}{\rho \mu_{d}}}-\mu_{r} e^{-\frac{\gamma}{\rho \mu_{r}}}}{\mu_{d}-\mu_{r}}\right)^{k}\right] d \gamma} \\
& =\frac{N \log _{2}(e)}{2 \rho\left(\mu_{d}-\mu_{r}\right)} \int_{0}^{\infty} \ln (1+\gamma)\left(e^{-\frac{\gamma}{\rho \mu_{d}}}-e^{-\frac{\gamma}{\rho \mu_{r}}}\right) \text {. } \\
& {\left[\sum_{k=0}^{N-1}\left(\begin{array}{c}
N-1 \\
k
\end{array}\right)\left(\frac{-1}{\mu_{d}-\mu_{r}}\right)^{k}\right. \text {. }} \\
& \left.\sum_{i=0}^{k}\left\{\left(-\mu_{r} e^{-\frac{\gamma}{\rho \mu_{r}}}\right)^{i}\left(\mu_{d} e^{-\frac{\gamma}{\rho \mu_{d}}}\right)^{k-i}\right\}\right] d \gamma \\
& =\frac{N \log _{2}(e)}{2 \rho\left(\mu_{d}-\mu_{r}\right)} \int_{0}^{\infty} \ln (1+\gamma)\left(e^{-\frac{\gamma}{\rho \mu_{d}}}-e^{-\frac{\gamma}{\rho \mu_{r}}}\right) \text {. } \\
& {\left[\sum_{k=0}^{N-1}\left(\begin{array}{c}
N-1 \\
k
\end{array}\right)\left(\frac{-1}{\mu_{d}-\mu_{r}}\right)^{k}\right. \text {. }} \\
& \left.\sum_{i=0}^{k}\left\{(-1)^{i} \mu_{r}^{i} \mu_{d}^{k-i} e^{-\left(\frac{i}{\rho \mu_{r}}+\frac{k-i}{\rho \mu_{d}}\right) \gamma}\right\}\right] d \gamma \\
& =\frac{N \log _{2}(e)}{2 \rho\left(\mu_{d}-\mu_{r}\right)} \sum_{k=0}^{N-1}\left(\begin{array}{c}
N-1 \\
k
\end{array}\right)\left(\frac{-1}{\mu_{d}-\mu_{r}}\right)^{k} \text {. } \\
& \sum_{i=0}^{k}\left\{( - 1 ) ^ { i } \mu _ { r } ^ { i } \mu _ { d } ^ { k - i } \left(\int_{0}^{\infty} \ln (1+\gamma) e^{-\left(\frac{i+1}{\rho \mu_{r}}+\frac{k-i}{\rho \mu_{d}}\right) \gamma} d \gamma\right.\right. \\
& \left.\left.-\int_{0}^{\infty} \ln (1+\gamma) e^{-\left(\frac{i}{\rho \mu_{r}}+\frac{k-i+1}{\rho \mu_{d}}\right) \gamma} d \gamma\right)\right\} \\
& =\frac{N \log _{2}(e)}{2 \rho\left(\mu_{d}-\mu_{r}\right)} \sum_{k=0}^{N-1}\left(\begin{array}{c}
N-1 \\
k
\end{array}\right)\left(\frac{-1}{\mu_{d}-\mu_{r}}\right)^{k} \text {. } \\
& \sum_{i=0}^{k}\left\{( - 1 ) ^ { i } \mu _ { r } ^ { i } \mu _ { d } ^ { k - i } \left[\mathbf{I}\left(\frac{i+1}{\rho \mu_{r}}+\frac{k-i}{\rho \mu_{d}}\right)\right.\right. \\
& \left.\left.-\mathbf{I}\left(\frac{i}{\rho \mu_{r}}+\frac{k-i+1}{\rho \mu_{d}}\right)\right]\right\} \text {. }
\end{aligned}
$$

where $\mathbf{I}(a) \triangleq \int_{0}^{\infty} \ln (1+\gamma) e^{-a \gamma} d \gamma=-\frac{1}{a} e^{a} E i(-a)$.

\section{REFERENCES}

[1] J. G. Proakis, Digital Communications. McGraw-Hill, Fourth ed., 2001.

[2] D. Tse and P. Viswanath, Fundamentals of Wireless Communication. Cambridge University Press, 2005.

[3] A. Sendonaris, E. Erkip, and B. Aazhang, "User cooperation diversity-Part I: System description," IEEE Trans. Commun., vol. 51, pp. 1927-1938, Nov. 2003.

[4] A. Sendonaris, E. Erkip, and B. Aazhang, "User cooperation diversity-Part II: Implementation aspects and performance analysis," IEEE Trans. Commun., vol. 51, pp. 1939-1948, Nov. 2003.

[5] J. N. Laneman, D. N. C. Tse, and G. W. Wornell, "Cooperative diversity in wireless networks: Efficient protocols and outage behavior," IEEE Trans. Inf. Theory, vol. 50, no. 12, pp. 3062-3080, Dec. 2004.

[6] H. Bolukbasi, H. Yanikomeroglu, D. D. Falconer, and S. Periyalwar, "On the capacity of cellular fixed relay networks," in Proc. IEEE CCECE, vol. 4, May 2004, pp. 2217-2220.

[7] N. Esseling, R. Pabst, and B. H. Walke, "Delay and throughput analysis of a fixed relay concept for next generation wireless systems," in Proc. European Wireless, 2005.

[8] T. Ohseki, N. Fuke, H. Ishikawa, and Y. Takeuchi, "Multihop mobile communications system adopting fixed relay stations and its time slot allocation schemes," in Proc. IEEE PIMRC, 2006.

[9] A. Bletsas, H. Shin, M. Z. Win, and A. Lippman, "Cooperative diversity with opportunistic relaying," in Proc. IEEE WCNC, vol. 2, pp. 1034-1039, Apr 2006.

[10] R. Knopp and P. Humblet, "Information capacity and power control in signle cell multiuser communications," in Proc. IEEE ICC, vol. 1, June 1995, pp. 331-335.

[11] P. Viswanath, D. N. C. Tse, and R. Laroia, "Opportunistic beamforming using dumb antennas," IEEE Trans. Inf. Theory, vol. 48, no. 6, pp. $1277-$ 1294, June 2002.

[12] S. M. Alamouti, "A simple transmit diversity technique for wireless communications," IEEE J. sel. Areas Commun. pp. 1451-1458, Oct 1998.

[13] R. Gozali, R. M. Buehrer, and B. D. Woerner, "The impact of multiuser diversity on space-time block coding," IEEE Comm. Lett, Aug. 2004.

[14] M. Hasna and M. Alouini, "A performance study of dual-hop transmissions with fixed gain relays," IEEE Trans. Wireless Comm., vol. 3, no 6, pp. 1963-1968, Nov. 2004.

[15] P. Larsson and N. Johansson, "Multiuser diversity forwarding in multihop packet radio networks," in Proc. IEEE WCNC, vol. 4, Mar. 2005, pp. 2188 2194.

[16] K. Navaie and H. Yanikomeroglu, "Induced cooperative multi-user diversity relaying for multi-hop cellular networks," in Proc. IEEE VTC, vol. 2, Spring. 2006, pp. 658-662.

[17] I. Hammerström, M. Kuhn, and A. Wittneben, "Channel adaptive scheduling for cooperative relay networks," in Proc. IEEE VTC, vol. 4, Fall. 2004 pp. 2784-2788

[18] Y. Ning, T. Hui, C. Shasha, and Z. Ping, "An adaptive frame resource allocation strategy for TDMA-based cooperative transmission," IEEE Commun Lett, vol. 11, pp. 417-419, 2007.

[19] S. Maheshwari, A. Boariu, Y. Saifullah, and Z. Li, "IEEE $802.16 \mathrm{~m} \mathrm{Re}-$ lay frame structure with legacy support," IEEE C802.16m-08/161, Mar. 16, 2008.

[20] J. N. Laneman and G. W. Wornell, "Distributed space-time coded protocols for exploiting cooperative diversity in wireless networks," IEEE Trans. Inf. Theory, vol. 49, pp. 2415-2425, Oct. 2003.

[21] Y. Xue and T. Kaiser, "Cooperated TDMA uplink based on hierarchically modulated alamouti code," in Proc. Int. Zurich Seminar on Commun, 2004. pp. 200-203.

[22] I. S. Gradshteyn and I. M. Ryzhik, Table of Integrals, Series, and Products. Academic Press, 2000.

[23] H. A. David, Order Statistics, 2nd ed. John Wiley \& Sons, 1980.

[24] M. Bala'zs, "Sum of independent exponential random variables with different parameters." [Online]. Available: http://www.math.bme.hu/ balazs /sumexp.html 


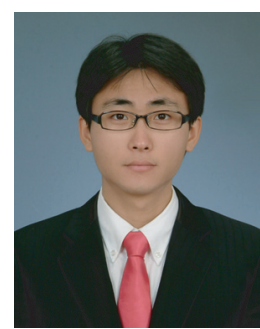

Min Suk Kang received the B.S. and the M.S. degrees in Electrical \& Computer Engineering from KAIST in 2006 and 2008, respectively. From February 2008, he is a research engineer with KAIST Institute for Information Technology Convergence, Daejeon, Korea. His research interests include MIMO cognitive radios, scheduling algorithms in relay networks, network coding, information theory, and link \& system-level simulations for wireless communication systems, such as IEEE $802.16 \mathrm{~m}$ and 3GPP LTE.

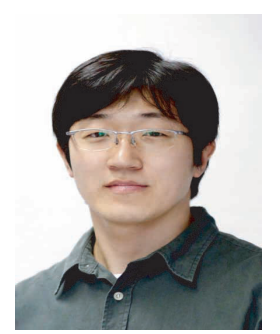

Bang Chul Jung received the B.S. degree in Electronics Engineering from Ajou University, Suwon, Korea, in 2002 and the M.S. and Ph.D. degrees in Electrical \& Computer Engineering from Korea Advanced Institute of Science and Technology (KAIST), Daejeon, Korea, in 2004 and 2008, respectively. From January 2008, he is a senior research engineer with KAIST Institutes for Information Technology Convergence, Daejeon, Korea. He received Bronze Prize in Intel Student Paper Contest in 2005. His research interests include orthogonal resource hopping systems for packet data transmission, OFDM systems, radio resource management, wireless scheduling algorithms, information theory, link \& system-level simulations for 4G wireless communication systems, adaptive modulation \& coding, and MIMO systems.

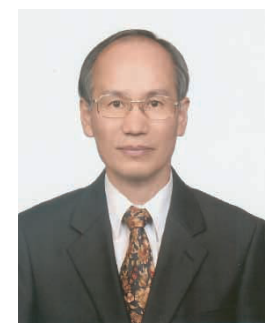

Dan Keun Sung received the B.S. degree in Electronics Engineering from Seoul National University in 1975 and the M.S. and Ph.D. degrees in Electrical \& Computer Engineering from the University of Texas at Austin, in 1982 and 1986, respectively. In 1986 he joined the faculty of KAIST where he is currently professor at the School of Electrical Engineering and Computer Science. He was director of the Satellite Technology Research Center (SaTReC) of KAIST from 1996 to 1999. He is editor of IEEE Communication Magazine. He was division editor of the Journal of Communications and Networks. His research interests include mobile communication systems \& networks with special interest in resource management, WLANs, WPANs, high speed networks, next generation IP based networks, traffic control in wireless \& wireline networks, signaling networks, intelligent networks, performance \& reliability of communication systems, and microsatellites. He received National Order of Merits, Dongbaek Medal in 1992, Research Achievement Award in 1997, MoMuc Paper Award in 1997, Academic Excellent Award in 2000, Best Paper Award in APCC2000, and This Month's Scientist Award by Ministry of Science and Technology (MOST) and Korea Science and Engineering Foundation (KOSEF) in 2004. He is a senior member of IEEE and a member of the National Academy of Engineering of Korea. 